\title{
A SIMPLE AND FAST MATLAB-BASED PARTICLE SIZE DISTRIBUTION ANALYSIS TOOL
}

\author{
JESUS D. ORTEGA ${ }^{1}$, IRMA R. VAZQUEZ ${ }^{1}$, PETER VOROBIEFF $^{1} \&$ CLIFFORD K. HO $^{2}$ \\ ${ }^{1}$ University of New Mexico, Albuquerque, NM, USA. \\ ${ }^{2}$ Concentrating Solar Technologies, Sandia National Laboratories, Albuquerque, NM, USA.
}

\begin{abstract}
Particle size distribution is one of the most important physical properties of a particulate sample. Traditional particle-sizing methods to estimate a geometrical particle size distribution employ a sieve analysis (or gradation test), which entails filtering the particles through a series of sieves and measuring the weight remaining on each sieve to estimate the number-weighted particle size distribution. However, these two quantities have the same value only if particles are perfectly spherical and round. On the other hand, a particle sizer such as the Malvern particle size analyzer, which uses laser diagnostics to measure the particle sizes, can be a hefty investment. Alternatively, imaging techniques can be applied to estimate the size of these particles by scaling a reference dimension to the pixel size, which in turn is used to estimate the size of the visible particles. The focus of this work is to present a simple methodology using a DSLR camera and an illuminated LED panel to generate enough contrast. Using the camera and lens properties, the scale, or size, of any image can be obtained based on the mounting distance of the camera with respect to the target. An analysis tool was developed in MATLAB where the images are processed automatically based on the prescribed camera and lens properties embedded within the same image file and requiring the user to only input the mounting distance of the camera. So far, results show a positive agreement when comparing to measurements using ImageJ imaging tools and a sieve analysis. Future tests will analyze different particle sizes and types, as well as using a Malvern particle size analyzer to corroborate the results.

Keywords:imaging methods, particle analysis, particle sizing.
\end{abstract}

\section{INTRODUCTION}

The estimation of an accurate particle diameter is critical for small- andlarge-scale calculations and experiments ranging from pharmaceutical unit operations [1], oil drilling [2], paints, metallic powders, agriculture, pollution control, and food products [3]. Throughout the applications, a narrow particle size distribution (PSD) is desired to produce higher manufacturability, thereby improving the product competence and quality [1]. The measured particle diameter value depends on particle-sizing methodology used as well as the particle morphology. Ways to measure particle size include laser-based systems [1], sieve analysis [5], X-ray methods [6], scanning electron microscope (SEM) [7, 8], among others.

However, more often than not, the particle shapes and sizes throughout samples are not necessarily the same. This difference in morphology can alter the final average particle diameter value which can impact experiments and limit applications. For example, the most commonly used method to assess a PSD is a sieve analysis (i.e. gradation), whichrelies on the ability for particles to flow through a set of metal meshes which is directly affected by the particle's sphericity and roundness $[4,8]$. Moreover, the resolution of the measurements is limited by the sieve sizes chosen, which can yield inaccurate results for narrow PSDs [9]. While the measurement is straightforward,there are many simplifying assumptions, such as 
assuming all the particles to be perfect spheres and assuming all the particles retained within every sieve step have an average size which are required to complete the assessment, potentially leaving out relevant particle morphology data. Aside from that, further limitation such as particle binding within the mesh will disturb particle flow, while limiting the measurements to dry particles only.

In a recent work by Ortega et al., the team showed the importance of utilizing the correct particle size which is necessary to estimate the mass flow rate egress of particles from a cavity-type falling particle receiver [10-11]. Furthermore, if the mass flow rate of particles egressing the cavity is known, the heat loss of the system due to particle egress can also be estimated [12]. Similarly, these particles can be used as tracers to estimate the total advective losses of the particle plumes egressing from the system comprised of air and particles.

In this work, a particle-sizing method using imaging techniques is presented. The MATLAB-based script methodology is able to provide (i) morphological robustness for the analyses,(ii) high accuracy and speedy tests, and(iii) a low-cost solution to the aforementioned challenges. The imaging method proposed in this work addresses these issues via an analysis tool developed in MATLAB, where the images are processed automatically based on the prescribed camera and lens properties embedded within the same image file. The practicality of this method will allow it to be more easily implemented in particle-sizing applications at a low cost with high accuracy and fast results. This method is compared to sieve analysis data and corroborated with ImageJ imaging tools as described in the following in detail.

\section{IMAGING METHOD}

The team developed a MATLAB-based method to analyze the size of particles using a small sample of particles which are imaged with sufficient contrast in the background. For the method development, a Nikon D3500 equipped with an AF-P DXNikkor 18-55 mm lens was used. The code requires a single image of the particles as well as some inputs which will be discussed in this section.

\subsection{Pre-analysis requirements}

Before an image is analyzed, there are four main inputs required: (1) the distance from lens to the target, (2) the focal length, (3) number of horizontal pixels, and (4) number of vertical pixels of the camera. The resolution of the camera chosen by the user will dictate the number of horizontal and vertical pixels. On the other hand, the distance from the lens to the target is a value that can be measured by the user as well as the focal length which is based on the camera configuration selected by the user.When these values are known, the size of the object can be calculated when the field of view (FOV) to focal length relationship is known following the example shown in Fig. 1.

This can be achieved by substituting the appropriate lens correlations between the angular field of view $(\theta)$ and the focal length $(f)$ into eqn (1), where $(X)$ is the corresponding spatial field of view and $(L)$ is the distance from the lens to the object of interest. For this study, the lens used was an AF-P DXNIKKOR 18-55mm f/3.5-5.6G which has characteristic focal length correlation [13]yield the generation of eqns (2) and (3) to correlate focal length to spatial field of view for the image and determine the correct image scaling. 


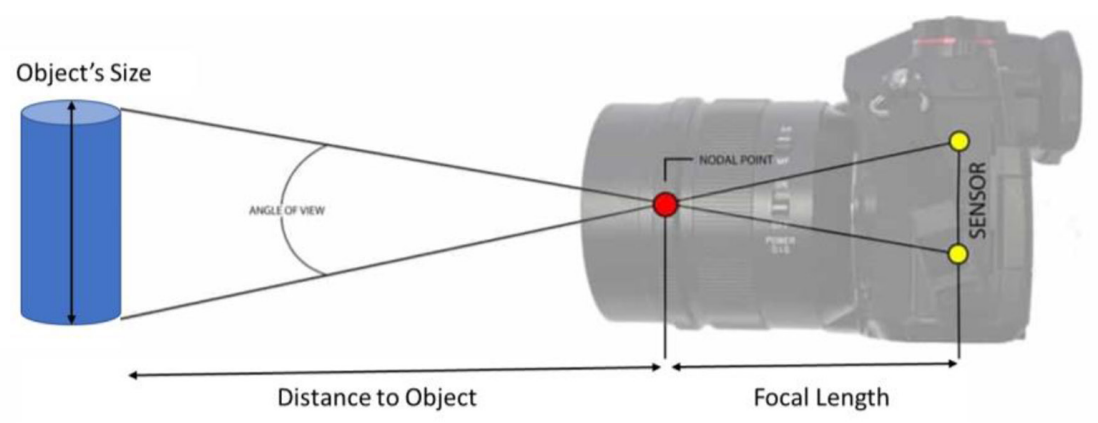

Figure 1: Schematic depicting the geometric relationship between the focal length, distance to the object of interest, and the object's size. Location of Nodal point can vary.

$$
\begin{gathered}
X=2 L \tan \left(\frac{\theta}{2}\right) \\
X_{f o v}=2 L \tan \left(\frac{461.785}{f^{0.908}}\right) \\
Y_{f o v}=2 L \tan \left(\frac{374.145}{f^{0.956}}\right)
\end{gathered}
$$

\subsection{Script methodology}

Once the pre-analysis requirements are fulfilled, the image will be loaded into MATLAB and scaled appropriately, which will lead to a final user input requirement. The script will require a region of interest (ROI) selection which allows the user to select the region within the image that will be used to complete the particle size calculation as shownin Fig.2.

Once the ROI is chosen, the image must be converted to an inverse binary image (see Fig. 2) in order to analyze the region properties using the built-in function regionprop in MATLAB. Applying this function enables the user to obtain properties from the current figure handle which can include area, centroid, circularity, Feret's diameters, major and minor axis lengths, perimeter, among other useful graphical properties. For this analysis, the team considers the equivalent diameter property to be appropriate as it computes the diameter from the measured area and perimeter of every particle.

To validate the particle size measurement using the in-house script, Image J, a powerful open-source platform to perform image analyses, was used to validate these measurements on the same ROIs within the image. The results shown in Table 1 show that that particle sizes estimated agree with those obtained from Image $\mathrm{J}$ which validates the methodology and move forward with the experimental validation.

\subsubsection{Particle cluster segmentation}

Particle clusters may form while handling particle samples sue to multiple factors; however, when dealing with PSDs, these particle clusters should be accounted for and ideally broken 
a)
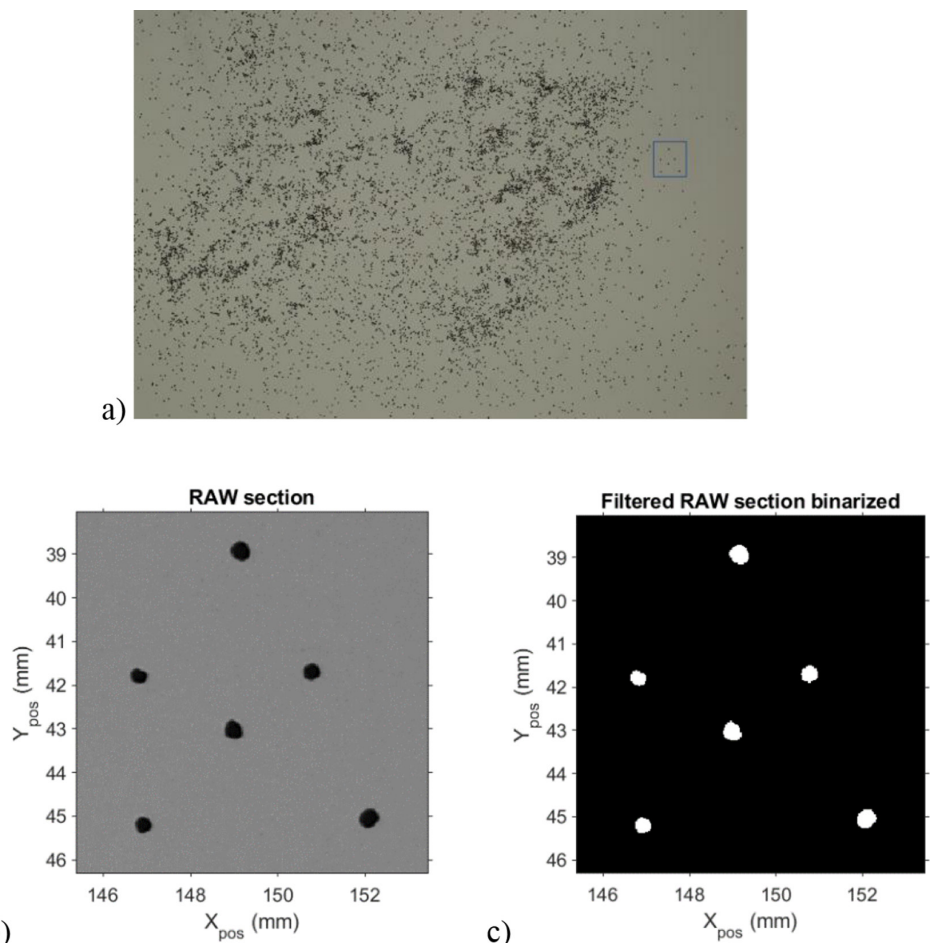

Figure 2: Selection of ROI from original image (a) yielding a cropped image as chosen by the user (b). The ROI is converted into an inverse binary image (c) with the particles shown in white and the background in black.

Table 1: Comparison of particle diameters estimated for Fig. 4 using Image $\mathbf{J}$ and the MATLAB script developed.

\begin{tabular}{llll}
\hline Particle & Area $\left(\mathbf{m m}^{2}\right)$ & $\begin{array}{c}\text { Image J } \\
\text { Diameter }(\boldsymbol{\mu m})\end{array}$ & $\begin{array}{l}\text { MATLAB } \\
\text { Diameter }(\boldsymbol{\mu m})\end{array}$ \\
\hline 1 & 0.158 & 448.5 & 445.0 \\
2 & 0.12 & 390.9 & 386.4 \\
3 & 0.104 & 363.9 & 361.4 \\
4 & 0.143 & 426.7 & 425.9 \\
5 & 0.153 & 441.4 & 440.3 \\
6 & 0.112 & 377.6 & 372.7 \\
\hline
\end{tabular}

apart either before or during processing. To ensure that the clusters of particles were not analyzed as one large irregular particle, a watershed segmentation technique was implemented to break the clusters apart [14].

Fig. 3 shows the results of the implementation of the watershed technique for the script developed. Applying this technique will help the segmentation of clusters and yield a more accurate representation of the PSD of the particle sample. 

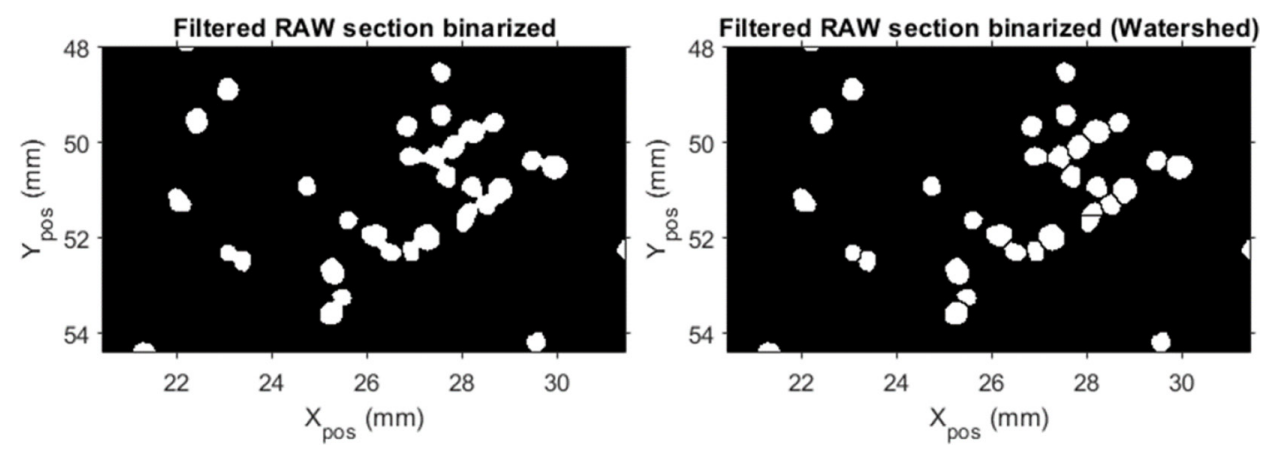

Figure 3: Comparison of binary images without (left) and with (right) a watershed technique applied to eliminate particle clusters.

\section{EXPERIMENTAL METHODOLOGY}

To evaluate the accuracy of the imaging method, the team collected particle samples for two different materials. First, Carbo HSP 40/70 a synthetic bauxite particle used in the oil and gas industry for fracking as well as solar particle receivers. This particle has very high sphericity and roundness coefficients, a narrow PSD (resembling mono dispersion) as well as being quite dark in color which makes it ideal for the analyses. On the other hand, sand has a very large PSD, colorrange, and shape variations, which places it on the other side of the spectrum for the experimental measurements.

\subsection{Camera experimental setup}

The team designed a camera rig which will hold the Nikon camera at a fixed position for all the measurements; similarly, to create sufficient contrast for the particles, an LED panel was installed as shown in Fig.4. This rig will ensure that the images are taken with a fixed distance, a constant focal length, and consistent illumination.

\subsection{Particle sieve analysis}

The team collected and measured three individual samples of each of the particle types and completed a sieve analysis to estimate the median particle diameter and the mean particle diameter of particle sizes for each sample as shownin Fig.5.

\subsubsection{Median particle diameter}

The quantity is often found by interpolating from a cumulative size distribution plot to obtain the 50th percentile size on the plot.

$$
X_{\text {median }}=D 50
$$

\subsubsection{Mean particle diameter}

There are multiple kinds of means which can take into account volume weighing and surface area of particles. The arithmetic mean $\left(d_{1,0}\right)$ is the weighted averaged diameter of the particle set. On the other hand, the Sauter mean $\left(d_{3,2}\right)$ is an estimate of the average particle size. It can be defined as the diameter of a sphere that has the same volume-to-surface area ratio as the 

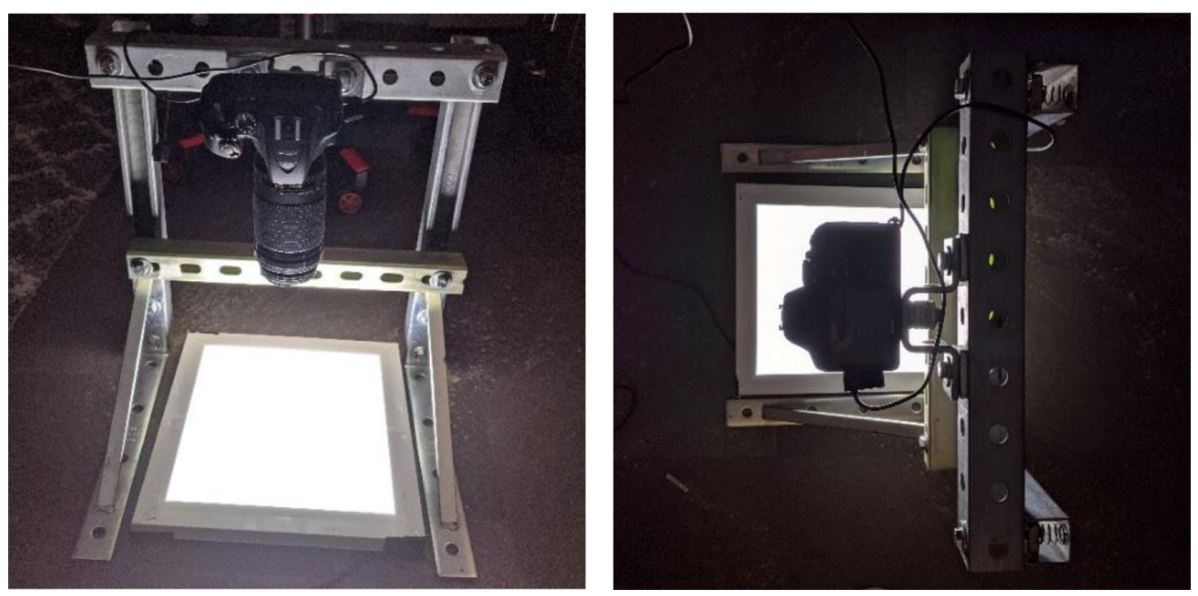

Figure 4: Left: Camera mounted on camera rig at $343 \mathrm{~mm}$ (13.5") from the LED panel. Right: Camera is mounted perpendicular to the LED panel which is cantered about the rig.
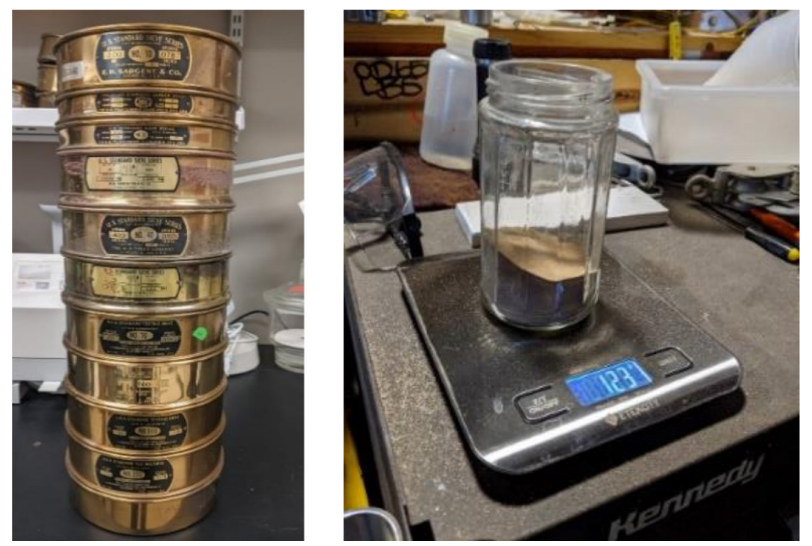

Figure 5: Left: Ten sieves from 75 to $2000 \mu \mathrm{m}$ were used for the sieve analysis. Right: The mass retained within every pan was measured using a scale with $\mathrm{a} \pm 1$ gr resolution.

particle analyzed as shown in eqn (5) [15]. It should be noted that this relationship assumes that a PSD for a poly-dispersed distribution has the same total volume and total surface area as a mono-dispersed distribution of the same particle.

$$
d_{p, q}=\left(\frac{\sum_{i=1}^{N} n_{i} d_{i}^{p}}{\sum_{i=1}^{N} n_{i} d_{i}^{q}}\right)^{\frac{1}{p-q}}
$$

\subsection{Imaging method}

Using the camera rig designed, the particles are dispersed along the LED panel to ensure that the formation particle clusters are reduced and can be seen by the camera as shownin 


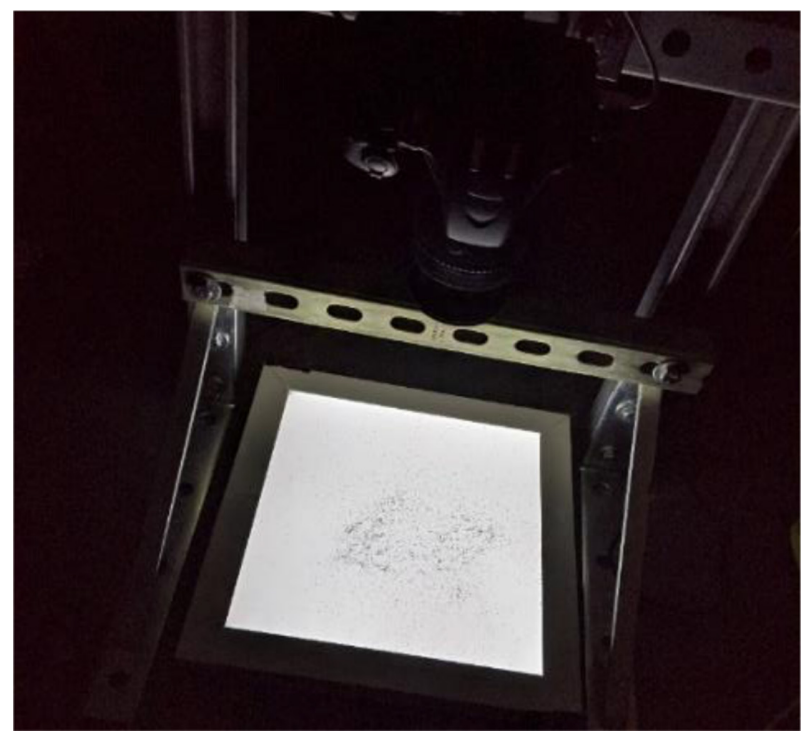

Figure 6: Camera rig with particles dispersed on the LED panel for imaging.

Fig. 6. It should be noted that for every sample that was collected for the sieve analyses, five sub-samples were taken for each sample to be imaged. Lastly, for every image captured, five different regions were analyzed to ensure consistency.

\section{EXPERIMENTAL RESULTS AND DISCUSSIONS}

\subsection{Sieve analysis}

The sieve analysis measurements followed the ASTM B214-07 testing procedure with sieve sizes ranging from 75 to $2000 \mu \mathrm{m}$. The cumulative size distribution curves for the Carbo HSP and sand particles can be observed in Fig. 7. These curves were generated for all three individual tests as well as for the combined total for all the tests. From thesedata, the median and the mean particle diameters can be calculated as shown in Table 2.

World oil presents a compilation of multiple proppants with properties. The report shows that CarboProp 40/70 has a median particle size of $324 \mu \mathrm{m}$, while the approximate median

Table 2: Measured median (D50), arithmetic mean (D $(1,0)$ ), and Sauter mean (D $(3,2))$ for the three sample tests of every particle type as well as the combined sample total.

\begin{tabular}{lllllll}
\hline \multicolumn{4}{c}{ Carbo particles } & \multicolumn{3}{c}{ Sand particles } \\
& $\begin{array}{l}\mathbf{D 5 0} \\
(\boldsymbol{\mu m})\end{array}$ & $\begin{array}{l}\mathbf{D}(\mathbf{1 , 0}) \\
(\boldsymbol{\mu m})\end{array}$ & $\begin{array}{l}\mathbf{D}(\mathbf{3}, \mathbf{2}) \\
(\boldsymbol{\mu m})\end{array}$ & $\begin{array}{l}\mathbf{D 5 0} \\
(\boldsymbol{\mu m})\end{array}$ & $\begin{array}{l}\mathbf{D}(\mathbf{1 , 0}) \\
(\boldsymbol{\mu m})\end{array}$ & $\begin{array}{l}\mathbf{D}(\mathbf{3}, \mathbf{2}) \\
(\boldsymbol{\mu m})\end{array}$ \\
\hline Test 1 & 313.1 & 374.7 & 396.2 & 386.3 & 516.0 & 974.4 \\
Test 2 & 334.6 & 407.5 & 438.5 & 395.7 & 530.7 & 960.1 \\
Test 3 & 332.1 & 405.9 & 433.9 & 372.2 & 486.7 & 917.0 \\
Combined & $\mathbf{3 2 6 . 1}$ & $\mathbf{3 9 7 . 1}$ & $\mathbf{4 2 5 . 4}$ & $\mathbf{3 8 3 . 9}$ & $\mathbf{5 1 1 . 3}$ & $\mathbf{9 5 1 . 1}$ \\
\hline
\end{tabular}



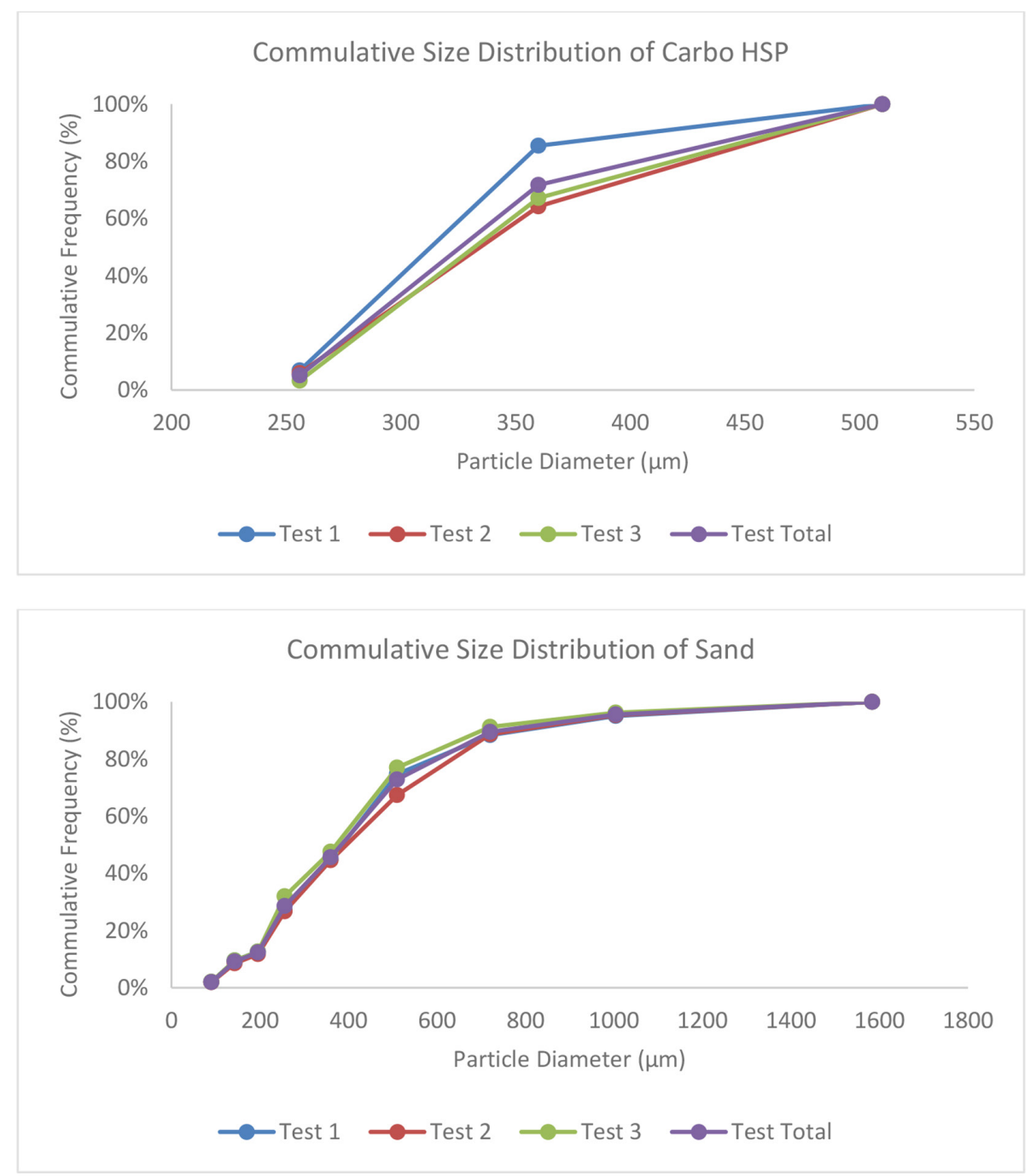

Figure 7: Cumulative particle size distribution of Carbo HSP 40/70 particles (top) and sand particles (bottom) for the three samples completed and the combined total.

from the total samples tested was $326 \mu \mathrm{m}$, which shows that the measurements performed align well with reported values [16].

\subsection{Imaging method}

As mentioned before, five sub-samples from the original three samples taken from both particle types are analyzed individually. For every image of the sub-samples, five regions are chosen near the four corners and the center of the image to complete the imaging analysis. Once the analysis was completed, a histogram of the size distribution is generated for the 75 cases analyzed per particle type as shown in Fig. 8.

The results of the study are summarized in Tables 3 and 4. It can be observed that for Carbo particles, the average and median diameters measured using the imaging method- 

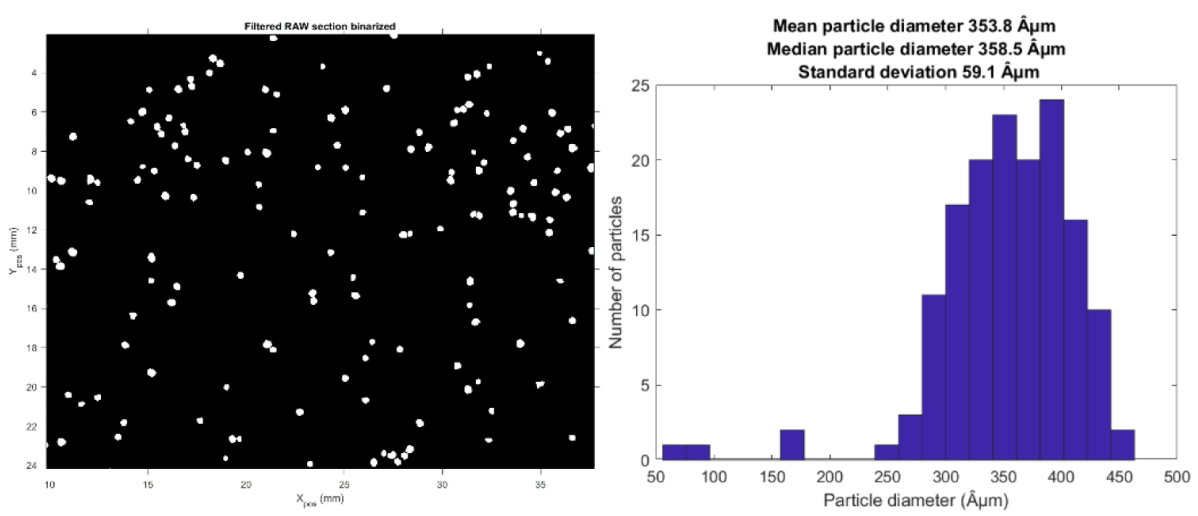

Figure 8: Sample image of a Carbo particle distribution with its corresponding particle size distribution plot. The outliers on the left correspond to some a small subset of particles considered as anomalies.

Table 3: Measured median (D50), arithmetic mean (D $(1,0))$, and Sauter mean (D $(3,2))$ for the three sample tests of the Carbo particle as well as the combined sample total compared to the average, median, and standard deviation of the particle diameters measured through the image method.

\begin{tabular}{lllllll}
\hline & \multicolumn{3}{c}{ Sieve analysis } & \multicolumn{3}{c}{ Imaging method } \\
& $\begin{array}{l}\mathbf{D 5 0} \\
(\boldsymbol{\mu m})\end{array}$ & $\begin{array}{l}\mathbf{D}(\mathbf{1 , 0}) \\
(\boldsymbol{\mu m})\end{array}$ & $\begin{array}{l}\mathbf{D}(\mathbf{3}, \mathbf{2}) \\
(\boldsymbol{\mu m})\end{array}$ & $\begin{array}{l}\mathbf{D}_{\text {avg }} \\
(\boldsymbol{\mu m})\end{array}$ & $\begin{array}{l}\mathbf{D}_{\text {median }} \\
(\boldsymbol{\mu m})\end{array}$ & $\begin{array}{l}\boldsymbol{\sigma}_{\text {diam }} \\
(\boldsymbol{\mu m})\end{array}$ \\
\hline Test 1 & 313.1 & 374.7 & 396.2 & 355.0 & 358.5 & 70.0 \\
Test 2 & 334.6 & 407.5 & 438.5 & 355.1 & 360.0 & 69.7 \\
Test 3 & 332.1 & 405.9 & 433.9 & 358.6 & 363.9 & 67.1 \\
Test total & $\mathbf{3 2 6 . 1}$ & $\mathbf{3 9 7 . 1}$ & $\mathbf{4 2 5 . 4}$ & $\mathbf{3 5 6 . 3}$ & $\mathbf{3 6 0 . 0}$ & $\mathbf{6 8 . 9}$ \\
\hline
\end{tabular}

Table 4: Measured median (D50), arithmetic mean (D $(1,0))$, and Sauter mean (D $(3,2))$ for the three sample tests of the Carbo particle as well as the combined sample total compared to the average, median, and standard deviation of the particle diameters measured through the imaging method.

\begin{tabular}{lllllll}
\hline \multicolumn{3}{c}{ Carbo particles } & \multicolumn{3}{c}{ Sand particles } \\
& $\begin{array}{l}\mathbf{D 5 0} \\
(\boldsymbol{\mu m})\end{array}$ & $\begin{array}{l}\mathbf{D}(\mathbf{1 , 0}) \\
(\boldsymbol{\mu m})\end{array}$ & $\begin{array}{l}\mathbf{D}(\mathbf{3}, \mathbf{2}) \\
(\boldsymbol{\mu m})\end{array}$ & $\begin{array}{l}\mathbf{D}_{\text {avg }} \\
(\boldsymbol{\mu m})\end{array}$ & $\begin{array}{l}\mathbf{D}_{\text {median }} \\
(\boldsymbol{\mu m})\end{array}$ & $\begin{array}{l}\boldsymbol{\sigma}_{\text {diam }} \\
(\boldsymbol{\mu m})\end{array}$ \\
\hline Test 1 & 386.3 & 516.0 & 974.4 & 183.5 & 154.4 & 133.0 \\
Test 2 & 395.7 & 530.7 & 960.1 & 169.6 & 140.3 & 115.8 \\
Test 3 & 372.2 & 486.7 & 917.0 & 174.4 & 148.7 & 110.5 \\
Test total & $\mathbf{3 8 3 . 9}$ & $\mathbf{5 1 1 . 3}$ & $\mathbf{9 5 1 . 1}$ & $\mathbf{1 7 5 . 8}$ & $\mathbf{1 4 7 . 5}$ & $\mathbf{1 1 9 . 8}$ \\
\hline
\end{tabular}


ology correspond to those measured with the sieve analysis. On the other hand, there is a large discrepancy in these values for the sand particles. The team believes that these discrepancies could be due to the morphology of the sand particles clogging the sieves during the analyses which will increase the mass of particles of smaller size to be retained on a larger step.

\subsubsection{Method constraints}

The team found that there are some artifacts generated during the process as shown in Fig. 11; however, their impact has yet to be characterized. These artifacts are generated from four different sources as shown in Figs. 9 and 10.

- The edge (i.e. cropped) effect of the regions selected can impact the size of the particles along the edge.

- The splitting effect yields individual particles broken into two different particles due to the morphology observed by the script.

- The center-splitting effect yields a smaller particle between two or more particles that were lumped together as observed by the script.

- The morphology effect impacts the way a single particle is identified, and due to the variations in light due to the translucency of the particle and the morphology, the script can interpret it as recognizing more than one particle.

While the team is aware of these effects, they have yet to be studied to determine their impact on the particle size estimation. However, a physical factor that could have an impact on the discrepancy of the sand particles could be the sieve clogs formed during testing.

\section{CONCLUSIONS AND FUTURE WORK}

A simple and accurate method to analyze particle size distributions was developed. The results showed great agreement with the Carbo particles which are more spherical and have a narrower PSD than sand particles. However, the results for the sand particles did not agree well, which could be due to multiple factors explained on the paper.

There are some artifacts that were generated on the images during the analyses; however, the team will need to further study their effects on the measurements. While these effects may or may not be trivial to the accuracy of the calculations in the words of R. Gonzalez and R. Woods authors of the widely used textbook (Digital Image Processing), they state that "segmentation of nontrivial images is one of the most difficult tasks in image processing. Segmentation accuracy determines the success or failure of computerized analysis procedures."

One current application for this analysis methodology was the characterization of the Carbo HSP 40/70 particles utilized at Sandia's falling particle receiver. While the supplier provides limited information on the particle morphology and size distribution, applying this methodology has allowed the team to verify the particle size used to estimate the mass egress and heat loss rates from the systems due to the advective losses experienced during operation.

To continue with this work, other particle-sizing techniques will be used to validate the results from the work presented. Similarly, the team would like to study other types of particles to ensure that this methodology is applicable for a wider range of particles, bubbles, and droplets. 

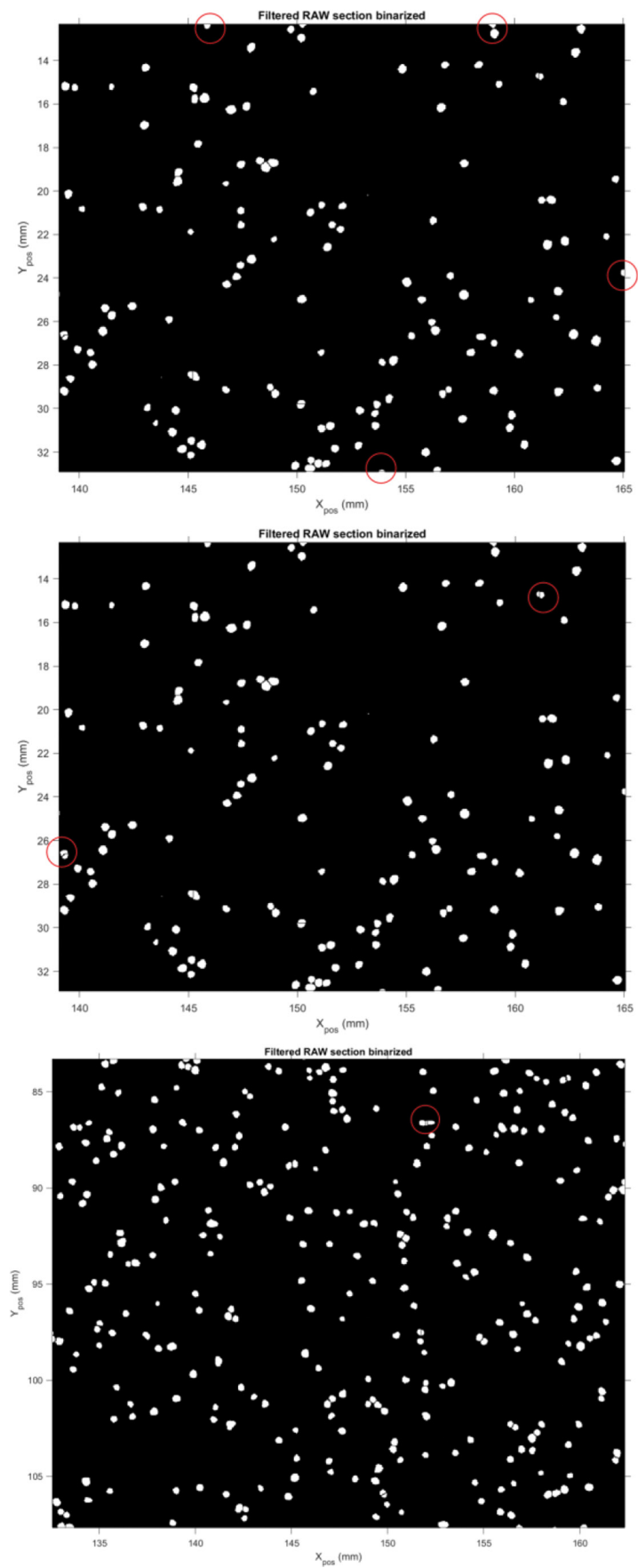

Figure 9: Three artifacts were observed during the image analyses of the Carbo particles applying the methodology developed. Top: Edge effect yielding smaller particles within a sub-region of the image analyzed. Middle: Splitting effect breaking particles apart which are interpreted as two particles by the script. Bottom: Centersplitting effect breaking a group of two particles into three by the script. 

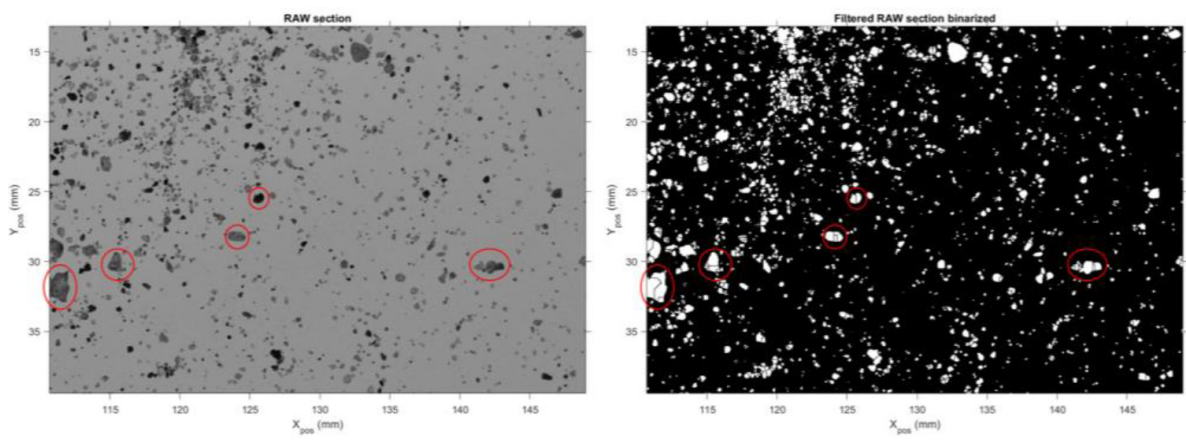

Figure 10: Besides the three artifacts observed on Fig.9, sand particles displayed a fourth artifact related to the morphology and translucency of the particles. This effect produces different shades which the script can interpret as separate particles.

\section{ACKNOWLEDGMENTS}

The authors thank Matthew Bauer and Andru Prescod from DOE for their management and support of this work, which was funded by DOE's Solar Energy Technologies Office (Award \#33869). Sandia National Laboratories is a multimission laboratory managed and operated by National Technology and Engineering Solutions of Sandia, LLC., a wholly owned subsidiary of Honeywell International, Inc., for the U.S. Department of Energy's National Nuclear Security Administration under contract DE-NA0003525. The authors also thank Dr. Linda Felton for allowing us to use her equipment for the sieve analysis.

\section{REFERENCES}

[1] Silva, Ana FT, et al., Particle sizing measurements in pharmaceutical applications: Comparison of in-process methods versus off-line methods.European Journal of Pharmaceutics and Biopharmaceutics, 85(3), pp. 1006-1018, 2013. https://doi.org/10.1016/ j.ejpb.2013.03.032

[2] Wajheeuddin, M. \& Enamul Hossain, M. An experimental study on particle sizing of natural substitutes for drilling fluid applications. Journal of Nature Science and Sustainable Technology, 8(2), pp. 1-14, 2014.

[3] Black, D.L., McQuay, M.Q. \& Bonin, M.P. Laser-based techniques for particle-size measurement: a review of sizing methods and their industrial applications.Progress in Energy andCombustion Science, 22(3), pp. 267-306, 1996. https://doi.org/10.1016/ s0360-1285(96)00008-1

[4] Fonseca, J., et al., Non-invasive characterization of particle morphology of natural sands.Soils and Foundations, 52(4), pp. 712-722, 2012. https://doi.org/10.1016/j. sandf.2012.07.011

[5] Alsaba, M., et al., Updated criterion to select particle size distribution of lost circulation materials for an effective fracture sealing.Journal of Petroleum Science and Engineering, 149, pp. 641-648, 2017. https://doi.org/10.1016/j.petrol.2016.10.027

[6] Yu, X., Y. F. \& Lu, S., Characterization of the pore structure and cementing substances of soil aggregates by a combination of synchrotron radiation $\mathrm{X}$-ray micro-computed tomography and scanning electron microscopy. European Journal of Soil Science, 68(1), pp. 66-79, 2017. https://doi.org/10.1111/ejss.12399 
[7] Karg, M.C.H., et al., Expanding particle size distribution and morphology of aluminium-silicon powders for Laser Beam Melting by dry coating with silica nanoparticles. Journal of Materials Processing Technology, 264, pp. 155-171, 2019. https://doi. org/10.1016/j.jmatprotec.2018.08.045

[8] Olson, E., Particle shape factors and their use in image analysis part 1: theory. Journal of GXP Compliance, 15(3), p. 85, 2011.

[9] Innopharma Labs, A Comparison of the Particle Sizing Techniques of Sieve Analysis and Eyecon. White Paper. Accessed: 6/2/2021. https://www.innopharmalabs.com/ sites/default/files/a_comparison_of_the_particle_sizing_techniques_of_sieve_analysis_and_eyecontm_mail_content.pdf

[10] Ho, C.K., et al., Characterization of particle and heat losses from falling particle receivers. Energy Sustainability. Vol. 59094. American Society of Mechanical Engineers, 2019.

[11] Ortega, J.D., et al., Particle Plume Velocities Extracted from High-Speed Thermograms through Particle Image Velocimetry. ASME 2021 15th International Conference on Energy Sustainability, ES 2021, 1-6.

[12] Ortega, J.D., et al., A Non-Intrusive Particle Temperature Measurement Methodology using Thermogram and Visible-Light Image Sets. ASME 2021 15th International Conference on Energy Sustainability, ES 2021, 1-6.

[13] Claff, B., Field-of-view of lenses by focal length. Nikonians. Camera Reviews, Lens Reviews. Online, https://www.nikonians.org/reviews/fov-tables. Accessed on: June 1 2021.

[14] Eddins, S., The Watershed Transform: Strategies for Image Segmentation. Technical Articles and Newsletters, MathWorks. Online, https://www.mathworks.com/company/ newsletters/articles/the-watershed-transform-strategies-for-image-segmentation.html. Accessed on: June 12021.

[15] An Introduction to Particle Characterization. AZO Materials, October 7, 2019. Online, https://www.azom.com/article.aspx?ArticleID=18502. Accessed on: June 22021.

[16] Proppant Tables/2015. WorldOil, Media, Carbo. Online https://www.worldoil.com/ media/3025/proppant-tables-2015.pdf. Accessed on: June 22021. 